\title{
Odour Based Human Identification and Classification using Neural Networks
}

\author{
Ahmed Qusay Sabri, Rayner Alfred
}

\begin{abstract}
Biometrics permits an individual to be authenticated and identified by computer systems following on a set of verifiable and identifiable data that are precise and unique in nature. This mechanism constitutes a cutting-edge method of identifying an individual since it precisely establishes more explicit and direct connection with humans than mere passwords since biometrics tend to use measurable behavioral and physiological characteristics of human. In this paper, a framework for human identification is proposed distinctively based on specific human odour features. 15 samples of female and male human odour are collected from different age groups, only 15 effective Volatile Organic Compounds (VOCs) are chosen. In this paper, several diverse functions of neural network activation are tested such as Levenberg-Marquardt backpropagation, Gradient descent backpropagation, and Resilient backpropagation. Besides, numerous neural network topologies are tested by means of variety hidden layers and different number of neurons and. Different energy functions were tested TAN-Sigmoid transfer, Linear transfer, and LOG-Sigmoid transfer. Considering the obtained results, employing two hidden layers with more neurons in the hidden layers- to be specific: 15 neurons in every layer- has yielded better accuracy in performance with an accuracy rate of 100\%. The unsurpassed framework for algorithm learning to be used for human identification can be backpropagation learning algorithm named the Levenberg-Marquardt. The best function for activation established in this paper is the function of TANSigmoid transfer. The performance accuracy consistency in recognizing human can be enhanced using a big number of study samples.
\end{abstract}

Index Terms: VOC, Odour, Resilient backpropagation, Gradient descent backpropagation, Levenberg-Marquardt backpropagation

\section{INTRODUCTION}

The human body produces VOCs that can be utilized to develop relevant information about the behavior, diseases, health status, and emotional state of a person. The odour of body is one of the human physical features that can be employed to identify different people. There are certain researches piloted to distinguish and categorize humans according to the body odour that is extracted from persons' armpits. An electronic nose, for instance, was invented with a sensing array and q system for data analysis [21]. Body odour recognition is viewed as a method of contact-less physical biometric which attempts to confirm the identity of a person. The body odour is extracted from several parts of the body existing in many forms such as armpits, exhalation, stools, farts, urine, or feet. Armpits can be one of the top sources for sampling some volatile chemicals that are released from human body. Armpit is indeed a body part where a huge number of bacteria and glands cooperate to release a strong scent which may produce a unique pattern that can assist in identifying different persons [32].

The Body odour of humans consists of numerous forms of components. Research conducted earlier has focused to identify the relevant Volatile Organic Compounds (VOCs) which can be utilized for detecting gender [5]. Twenty samples of male and female human odour were then collected with 15 out of 198 (VOCs) selected from each person using both entropy and Chi-square test for detecting and classifying gender via artificial neural networks. Diverse types of functions of neural network activation were then tested (e.g., Resilient backpropagation, Gradient descent backpropagation and Levenberg-Marquardt backpropagation) and numerous different topologies of neural network are tested with variations of neurons and hidden layers. Based on those relevant (VOCs) Volatile Organic Compounds, the proposed gender detection system produces accuracy percentage of $94.7 \%, 94.6 \%, 93.4 \%$ and with 4 layers/30 neurons, 3 layers/30 neurons and 2 layers/20 neurons respectively [6]. The factor of gender is one of the established demographic human being attributes; beside from gender there are many other demographic attributes such as ethnicity and age, which is identified via computer vision and is applied to several applications such as demographic studies, surveillance, human computer interaction, and biometrics $[3,7,18]$. I addition, metabolic body changes are occasionally associated with certain odour s that can be measured on sweat, breath or other excreta from individuals. The odour of human body comprises diverse types of volatile organic compounds that can be employed as diseases biomarkers. Experiments with trained dogs revealed that they can smell diseases such as hyperglycemia, hypoglycemia and cancer. However, no studies have been conducted to explore the natures of (VOCs) Volatile Organic Compounds which are smelled or used by these trained dogs (which ingredient is linked to each disease). If we could know the specific odour components of specific diseases, we would create artificial noses as systems for automated odour detection. An electronic nose system (e-nose)

Revised Manuscript Received on August 19, 2019.

Ahmed Qusay Basri, University of Sharjah, Sharjah, UAE.

Rayner Alfred, Knowledge Technology Research Unit, Universiti Malaysia Sabah, Kota Kinabalu, Sabah, Malaysia 
can be a mechanical instrument to decide the volatile compounds. The number of applications of e-nose has grown rapidly in the medical field. Some of those applications are diagnosis of urinary tract infection, the mycobacterium tuberculosis detection in sputum cultures and samples, diabetes and schizophrenic patients' identification, bacteria grouping, and exhaled breath analysis of several diseases such as asthma and lung cancer [17]. This is among many reliable biometric traits, like face, which is a very popular and has an accessibility reputation. However, the malicious environments, unfortunately, enable some attackers to create photocopies easily and beat or spoof these systems of face recognition. Spoofing stands as an effort to acquire authentication into a biometric system via presenting a kind of forgery evidence for a valid user. This face recognition vulnerability has induced serious attention from the biometric community with several papers on countermeasure studies being published [4]. The issue of soft biometric traits classification related to age, ethnicity, gender, and odour has not yet been a resolved due to problematic exposure conditions $[13,19]$. The biometric benefit is that it doesn't lose or change. Many personal characteristics, imaging methods, and body parts have been utilized as biometric systems such as teeth, ears, fingers, voices, hands, feet, eyes, gaits, veins, typing styles and signatures. Each biometric takes its own limitations and strength, so that each biometric is employed in applications of Identification (authentication) [2].

In this current research, the test of Gas Chromatograph Mass Spectrometry (GC/MS) is applied on Males and Females over different days to arrive at more consistent type of VOCs (Volatile Organic Compounds) emitted and extracted from the odour. We reached the most accurate, rigid, and stable list of VOCs from people with specific name of Gas, acquiring the result of a shortlist of 15 VOCs employed for the process of human identification. The purpose of this paper is to examine the Artificial Neural Network (ANN) performance in categorizing human in different settings based on the human's emission of VOCs. The settings stated include various topologies and activation functions for human detection where 15 VOCs are considered and collected as a neural networks input. These VOCs produced by human are inputted into the ANN where three functions of different activation are being tested, which are resilient backpropagations, Gradient descent and Levenberg-Marquardt. Several topologies for neural network are tested with variations of number of neurons and hidden layers as well. The remaining of the paper proceeds as follows; firstly, some related works on human identification and human VOC's roles will be highlighted. Secondly, the processes involved in human identification and Classification are described. Next, the results obtained will be analyzed and discussed. Finally, the research conclusion is presented by offering some future work suggestions which can be constructed upon the obtained results in this research.

\section{RELATED WORKS}

Humans are observed to have distinctive scents like a fingerprint or signature. The axillary region plays a significant role in producing individual odour [20, 21]. A system for odour sensing through various sensors with a technique for pattern recognition and with partially overlapping particularities has been examined [30]. Odour classification in dynamically varying concentration can be beneficial in various applications such as environment monitoring, fire detection, etc. However, odour classification reliant on transient sensor responses has been conducted so far under the constant odour concentration condition [30].

Neural networks $(\mathrm{NN})$, used to recognize patterns and to scrutinize multipart data, are displaying promising outcomes in chemical vapor recognition. They have become a significant method in the field of recognizing electronic nose pattern. Back-propagation network (BP) with its different form is the most beneficial NN pattern [31].

Research has been successful in identifying two different people through detecting human armpit odour by means of desktop electronic nose (E-nose). It has been further found that applying deodorant does not have any effective element on the relative identification of those two different people. However, throughout the experiment, the volunteer persons were asked to control their daily ordinary activities and lifestyle to behave averagely in a similar way on agreed guidelines. In practice, these guidelines and rules are considered difficult to be followed in most real-life world situations and applications. Furthermore, desktop E-nose is rather big and involves consuming more power that makes it problematic to be used in sampling process. An original E-nose that is handheld is more desirable as it can be utilized to recognize and detect human odour in the armpit body part. The E-nose produced can operate through a Wi-Fi or local area network with low power consumption option [32].

Another study was conducted to examine the facility of an e-nose to sense changes in the odour of human body of patients with diverse levels of renal dysfunction as compared with healthy persons. All healthy subjects $(n=11)$ managed to completely be distinguished from renal failure patients $(n=62)$ applying quadratic discriminant analysis and principal component analysis (PCA). The discrimination among study subjects with chronic renal failure and with end stage renal failure $(n=42)$ was successful at a rate of $92.5 \%$. Primary results of a clinical pilot study discovered a classification rate at $100 \%$ among primed alcoholic addicted patients, liver cirrhosis patients, and healthy subjects. The study objective was to apply e-nose system to diagnose heart failure through identifying disease-related sweat volatile gases produced by the skin surface and triggered by an impaired metabolic [17]

Efficient and accurate results obtained for fingerprint detection and face recognition using artificial neural net $[2,8$, $11,12,15]$. Palm print and biometric hands detection employing back-propagation neural net with Levenberg-Marquardt training algorithm used in the classification of Palm print Biometrics. 
The results obtained revealed that the most accurate classification can be attained at $99.9 \%$ using 2 hidden layers $[9,10]$. Consequently, in this study, the training algorithm of Levenberg algorithm is adopted as one out of three neural net algorithms. Employing neural net in the field of biological informatics is extremely effective. In fact, neural net comprises 3 hidden layers, input and output layers using back-propagation algorithm with gradient descent that is employed for ID identification to categorize subjects with a classification accuracy rate at 97\% [22]. Besides, a structure with multi-layer neural net using Levenberg algorithm was utilized as training algorithm for checking weights update of the hepatitis diseases diagnosis neural net. The results revealed a classification accuracy at a rate of $91.87 \%$ [23]. Classification of heart and brain biometrics by artificial neural network has an accuracy at a rate of $92.4 \%$ to $95.1 \%$ [24].

The main advantages of our research in this paper compared to previous works are using specific 15 gas list to identify the human, the accuracy percentage obtained is the best among other researches, the names of gases are declared and specified clearly, using new technology to collect the emitted gases from human, and overlap feature selection with more than one algorithm to support and simplify the classifier task.

\section{Human Identification Process Based ON ODOUR}

\section{A. Gas Chromatograph Mass Spectrometry Test (GC/MS) and Sweat Collection}

Study participants will be tested in consecutive days during the week with different genders and age group considered. They will go through a seven-day washout period before the test program during which they are permitted to only wash their armpit with a kind of neutral soap. They need to be present at the lab every morning to wash as a standardized procedure. They will be asked to wear a cotton $t$-shirt with pads under the armpits for a period of 4 hours during which they must adhere to their normal day activities. To have the best odour representation, study subjects are requested to run up and down the stairs for approximately 3 times with 3 minutes per time round. The individuals are asked to bring the cotton pads to the lab after 4 hours.

A sweat sample is presented to a Nalophan bag of $1.5 \mathrm{~L}$ nitrogen. The pad is then heated for 30 minutes up to $90^{\circ} \mathrm{C}$. Subsequently, the headspace will be collected from that Nalophan bag of every sample into the thermal desorption tube, namely Tenax/Carbograph, so that it is up to $1000 \mathrm{ml}$ total volume. Moreover, one more thermal desorption tube that has no sample and is under the same conditions of sampling was placed into nalophan bag that was used in sampling process. These study tubes were blanks; this is done to detect all compounds in the sweat samples. Fig. 1 illustrates a test result extracted from one person for all VOCs that were emitted from human sweat. During the study, 15 persons of different genders and ages were tested. The $\mathrm{X}$ axis signifies the number of minutes needed for the sample in the stated VOC detection device, whereas Y axis represents the detected VOC concentration. Table 1 summaries a list of 15 VOCs designated out of 198 gas that was emitted from study subject and will be used as an input data to the artificial neural network NN for the stated human identification target [16].

\section{B. Artificial Neural Network}

The task of classification is one of the most vital ones in artificial neural networks (ANN) applications [1]. Some kinds of batch training algorithms can be employed to train a certain network. Training algorithms types are three in number and are assessed for classification [1]. The algorithm of gradient descent backpropagation is a local search procedure of gradient descent. It is intended to measure the procedure output error and calculates the error gradient by regulating the weights in the descending gradient algorithm direction [1]. The algorithm of resilient backpropagation training works to eliminate the partial derivatives' magnitudes effects. This derivative sign is utilized to govern the derivative magnitude and the weight update direction and does not affect the weight update. The weight change size is decided by a distinct update value. The update value in every bias and weight is typically increased by a certain factor whenever the performance function derivative of that weight contains the same mark for two successive iterations [1].

Table 1. Selected gases to be used for person detection (15 Gas)

\begin{tabular}{cc}
\hline No & VOCs \\
\hline 1 & Acetaldehyde $\left(^{*}\right)$ \\
2 & 2-Propenal \\
3 & Methacrolein \\
4 & Furan \\
5 & Furan, 2-pentyl- \\
6 & Acetone \\
7 & 2-Butanone \\
8 & 3-Penten-2-one \\
9 & 2-Pentanone \\
10 & Furan, 2-pentyl- \\
11 & 5-Hepten-2-one, 6-methyl- \\
12 & Ethanol \\
13 & Propanal, 2-methyl- \\
14 & Butanal, 3-methyl- \\
15 & 2,3 Butanedione \\
\hline
\end{tabular}




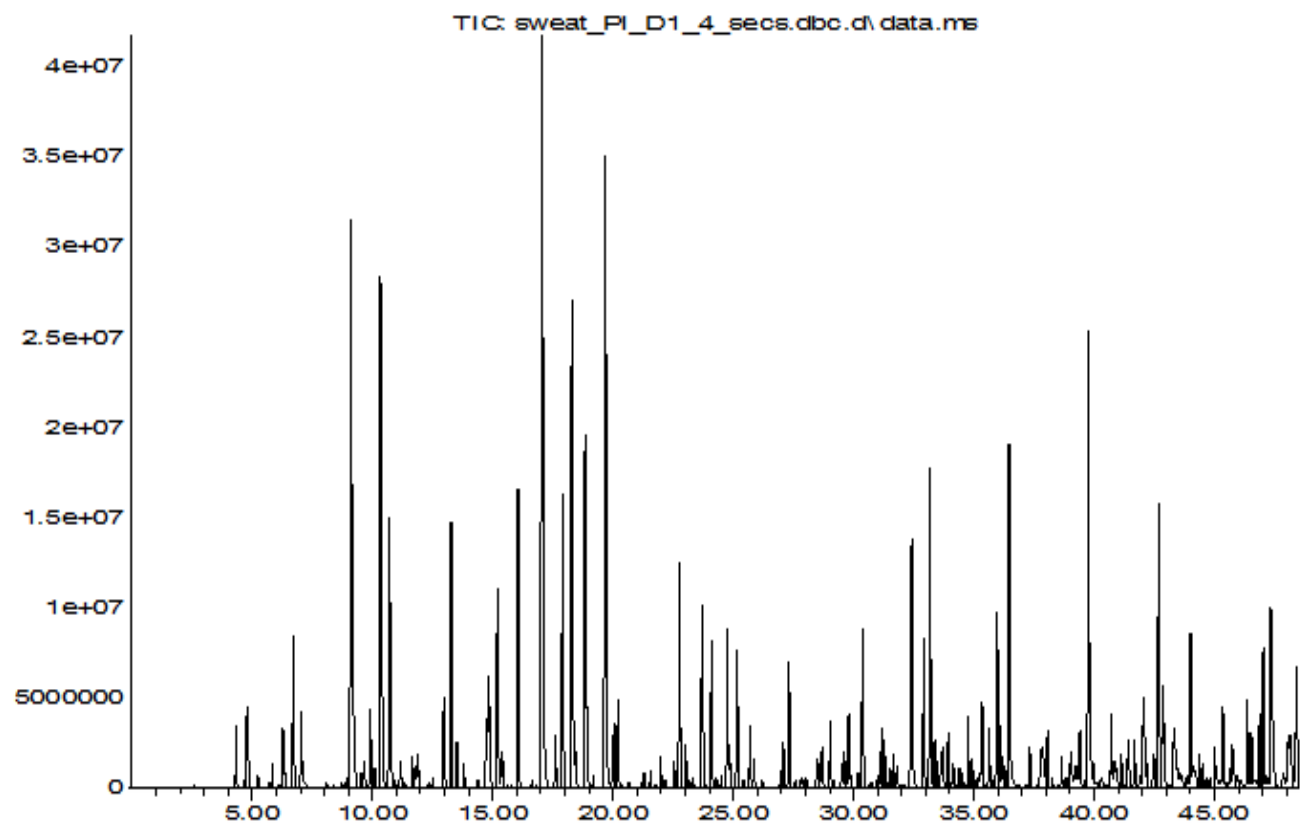

Fig. 1.All gases emitted from one person (198 Gas)

The algorithm of Levenberg-Marquardt backpropagation traces the multivariate function minimum which can be given as the squares sum of real-valued non-linear functions. This is an iterative method that operates so that performance function is always reduced in every algorithm iteration [1]. The backpropagation neural network acquires data by calculating the output layer errors to spot the errors in the intended hidden layers. By adopting this qualitative ability, this makes it decidedly fit to be applied on issues where no relationship can be established between the inputs and the output. The ANN techniques stand as very convenient in application domains to explain extremely nonlinear phenomena [14]. Because of its high rate of learning capabilities and plasticity, it has proved to be fit to be successfully executed within a wide range of applications [25, 29]. The association between the input $X$ and the neural network output Y is specified by [29]:

$$
\gamma_{t}=\omega_{o}+\sum_{j=1}^{q} \omega_{j} \cdot f\left(\omega_{0, j}+\sum_{i=1}^{p} \omega_{i, j} \cdot x_{t}\right)
$$

with $w_{j}(j=0,1,2, \ldots, q)$ and $w_{i, j}(I=0,1,2, \ldots, p ; j=1,2$, $\ldots, \mathrm{q})$ as the connection weights. $\mathrm{q}$ is the hidden nodes number, $\mathrm{p}$ is the input nodes number, and $f$ is a function for nonlinear activation which makes it possible for the system to absorb nonlinear features. In fact, the most widely implemented activation function regarding the output layer is the hyperbolic and sigmoid functions [29]. Since the purpose of this paper is to examine the varying effects of parameters settings employed for Human identification, several four settings are implemented as illustrated in Fig. 2 via 4, where the output neurons begin from 4 neurons, 6 neurons, 8 neurons and 10 neurons to identify the person using different pattern. These four settings consist of different topologies using 15 inputs, 2/3/4 hidden layers having 15 neurons each and 4/6/8/10 neurons output layer as shown in Fig.2 - Fig. 7. In every topology, three learning schemes will be adopted in the ANN; those learning algorithms are Resilient backpropagation, Levenberg-Marquardt backpropagation, and Gradient descent backpropagation. To arrive at the best results, 3 different activation functions were tested: Linear transfer, TAN- Sigmoid transfer, LOG- Sigmoid transfer function.

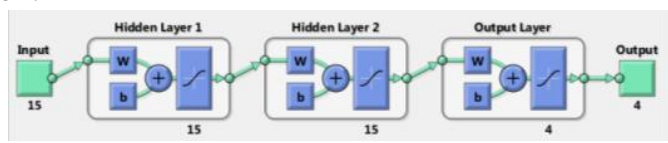

Fig. 2. 15 inputs, 2 hidden layers with 15 neurons each and 4 neurons output layer

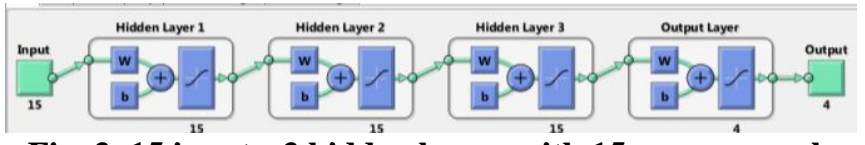

Fig. 3. 15 inputs, 3 hidden layers with 15 neurons each and 4 neurons output layer

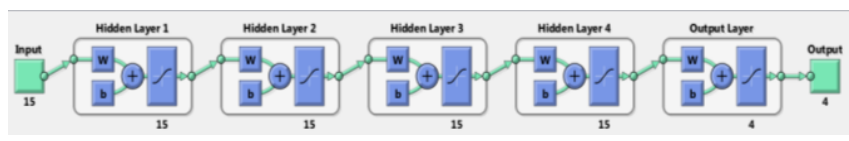

Fig. 4. 15 inputs, 4 hidden layers with 15 neurons each and 4 neurons output layer

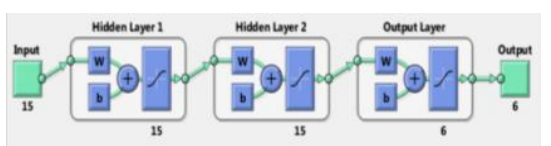

Fig. 5.15 inputs, 2 hidden layers with 15 neurons each and 6 neurons output layer

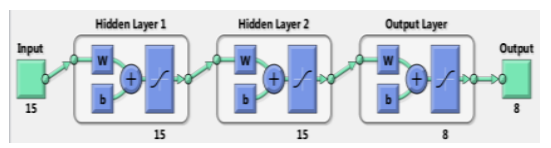

Fig. 6. 15 inputs, 2 hidden layers with 15 neurons each and 8 neurons output layer

Published By:

Blue Eyes Intelligence Engineering \& Sciences Publication 


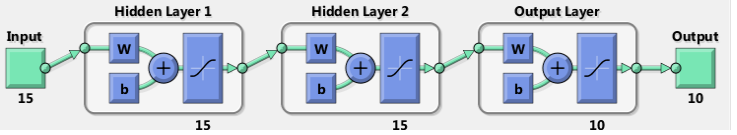

Fig. 7. 15 inputs, 2 hidden layers with 15 neurons each and 10 neurons output layer

\section{C.Levenberg-Marquardt backpropagation}

The algorithm of Levenberg-Marquardt (LM) is considered a variation of the known Newton's method which was originally designed to minimize the functions that are squares sums of different nonlinear functions, which is appropriate to the algorithm of neural network training where the index of performance is the mean squared error [23, 27]. The Levenberg approximates the Newton method employed to train ANNs. It is an optimization technique that can be more controlling than standard Backpropagation Neural Network (BPNN). Levenberg algorithm is very effective and fast having good global convergence property [28].

\section{D.Gradient Descent Backpropagation}

This feature is utilized to progressively, but constantly, decrease the error of output by correcting the weights. The biases and weights are then updated in the negative gradient direction of the performance functions. The feature of Backpropagation is implemented to estimate performance derivatives relating to the bias and weight variables. Regarding multi-layer networks, it is noticed that the relationship between any weights and the errors in the network requires calculation.

$$
\Delta \omega_{k}=-\alpha_{k} \cdot g_{k}
$$

where, $g_{k}$ is the current gradient, $\Delta \omega_{k}$ is a weights changes vector, $\alpha_{k}$ is the rate of learning that regulates the weight update length [29], which comprises propagating error from output nodes backwards through the internal layers of the network; one layer at a time, every layer error is then computed for every node. The computations of gradient descent backpropagation are illustrated below [29]:

- Calculate the signals of feed-forward from the input to the output

- Calculate output error e:

$$
\mathrm{e}=\frac{1}{2} \sum_{k}\left(T_{k}-O_{k}\right)^{2}
$$

where: $T_{k}$ : The network output vector and $O_{k}$ : The vector of the desired output.

- Backpropagate the signals of error by weighting it by the weights in the gradients of the related activation functions and previous layers.

- Calculating the parameters gradients regarding the feedforward signals stemming from the inputs and the signal of the backpropagated error.

- Update the parameters via the calculated gradients.

\section{E.Resilient Backpropagation}

This is considered the top algorithm that is measured in terms of accuracy, robustness, and convergence speed. As for training parameters, the algorithm of resilient backpropagation, it has regularly more capability to escape from local minima and gives faster convergence [26, 29]. It is only the partial derivative sign that is considered to govern the weight update direction that is multiplied by the size of the step. The resilient backpropagation weights update is as follows [29]:

$$
\Delta \omega_{k}=-\operatorname{sign}\left(\frac{\Delta E_{k}}{\Delta \omega_{k}}\right) \cdot \Delta_{k}
$$

\begin{tabular}{|c|c|c|c|}
\hline Algorithms & $\begin{array}{l}\text { Output } \\
\text { (Neurons) }\end{array}$ & $\begin{array}{l}\text { Hidden } \\
\text { Layers }\end{array}$ & $\begin{array}{c}\text { Best } \\
\text { detection } \\
\text { achieved }\end{array}$ \\
\hline Levenberg-Marquardt & $4,6,8,10$ & $2,3,4,5$ & $100 \%$ \\
\hline $\begin{array}{l}\text { Gradient Descent } \\
\text { Backpropagation }\end{array}$ & 4 & $2,3,4$ & $93 \%$ \\
\hline $\begin{array}{c}\text { Resilient } \\
\text { Backpropagation }\end{array}$ & 4 & $2,3,4$ & $87 \%$ \\
\hline
\end{tabular}

Table 2. Selected gases used for person detection (15 Gas)

Note: Input (Neurons) $=15$,

Activation Function $=$ TAN,Sigmoid, LIN

The purpose of using different output layers in Levenberg-Marquardt using 4, 6, 8, 10 neurons is that Levenberg-Marquardt produces the best performance in 4 neurons output layer, hence proving the stability and rigidity of neural network for a much bigger structure and higher number of persons, and only 4 neurons output tested for gradient descent backpropagation and Resilient backpropagation based on results obtained less than Levenberg-Marquardt. Starting with 4 neurons output layer for all neural networks based on available samples of 15 persons.

\section{RESULTS AND DISCUSSION}

Gradient Descent Backpropagation neural network is tested using 3 different topologies based on hidden layers, 2 hidden layers with 15 neurons result $64 \%$ accuracy, while 3 hidden layers each with 15 neurons results $73 \%$ accuracy, 4 hidden layers with 15 neurons each results $93 \%$ accuracy, Fig. 8 and 9 illustrate the obtained results. The best result obtained from 4 hidden layers with 15 neurons each results $93 \%$ accuracy will be tested using different activation function SIG and LIN for performance evaluation, the best result is TAN function with $93 \%$ accuracy while SIG function produce $73 \%$ and LIN produce $60 \%$ (Fig. 9). The purpose of getting such results may refer to this function is not suitable for biological data.

Published By: 


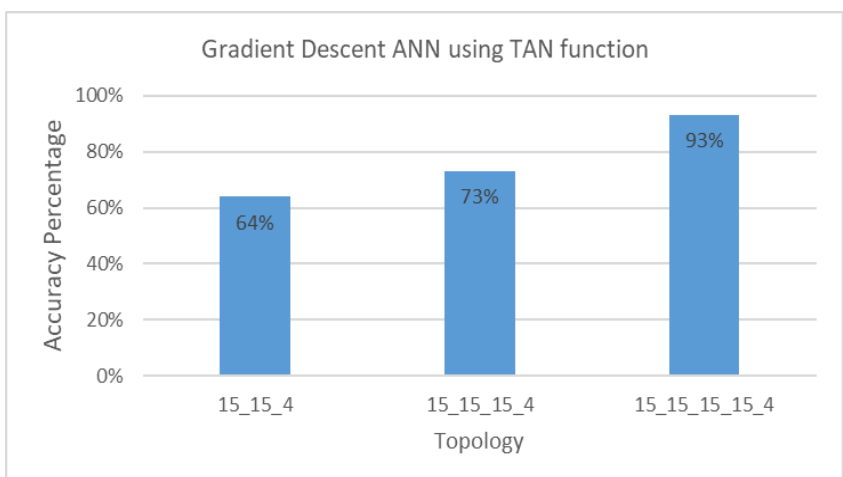

Fig. 8.Gradient Descent 3 different topologies accuracy using TAN function

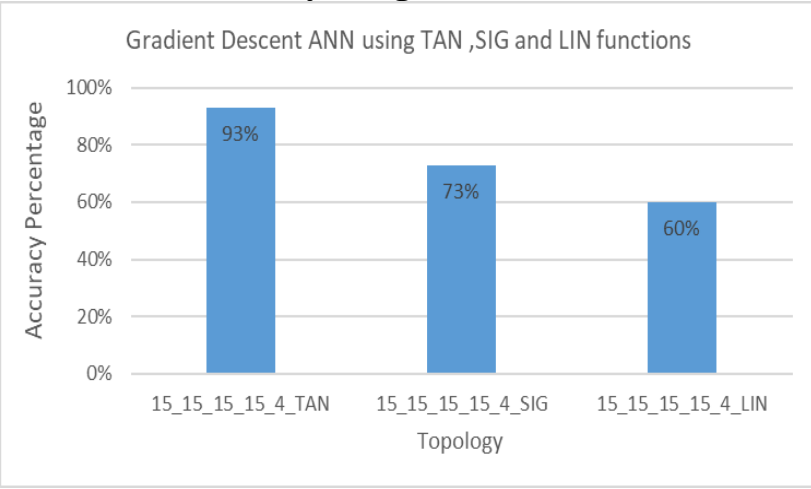

Fig. 9.Gradient Descent topology using TAN, SIG and LIN functions.

Resilient Backpropagation neural network tested using 4 different topologies based on hidden layers, 2 hidden layers with 15 neurons result $87 \%$ accuracy, while 3 hidden layers each with 15 neurons results $80 \%$ accuracy, 4 hidden layers with 15 neurons each results $87 \%$ accuracy, 5 hidden layers with 15 neurons each results $80 \%$ accuracy, Fig. 10 shows the obtained results. Actual calculations shown accuracy $87 \%$, Person9 and Person10 did not get detected, the result of this topology is the maximum performance of Resilient Backpropagation Neural Network. The best result is obtained from 2 hidden layers with 15 neurons each results $87 \%$ accuracy will be tested using different activation function SIG and LIN for performance evaluation, the best result is TAN function with $87 \%$ accuracy while SIG function produce $80 \%$ and LIN produce $60 \%$, Fig. 11 shows the obtained results. The purpose of getting such results may refer to this function is not suitable for biological data.

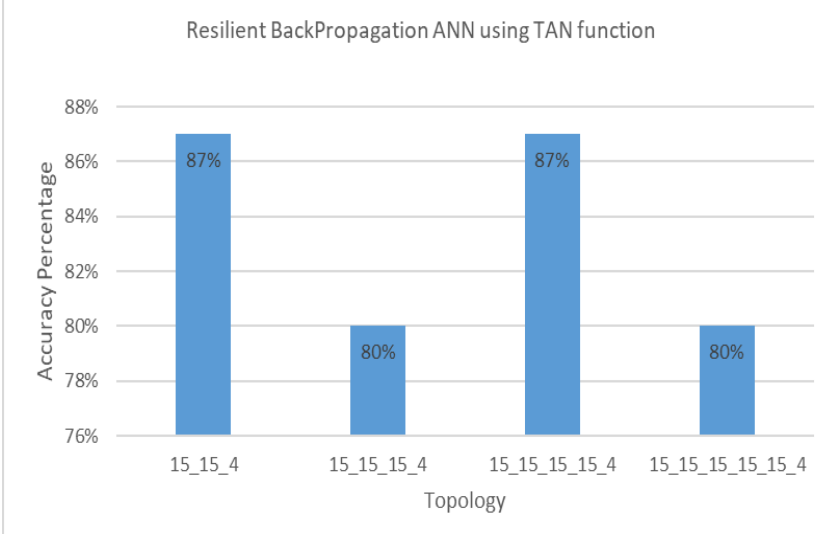

Fig. 10. Resilient Backpropagation with 3 different topologies accuracy using TAN function.

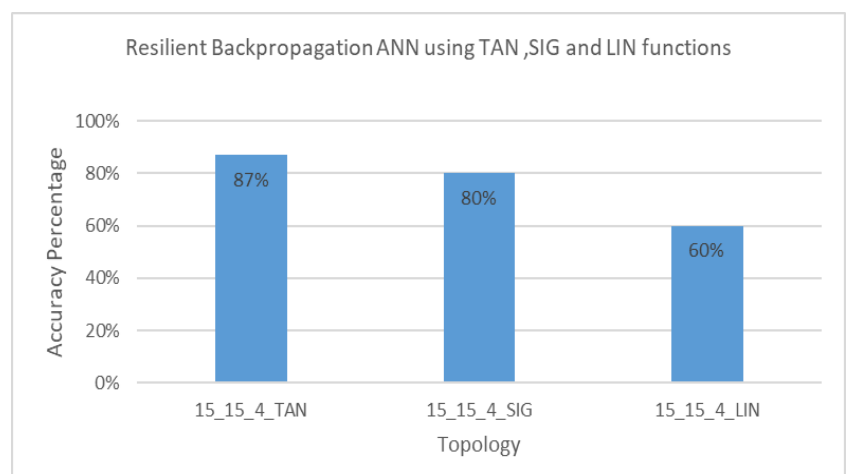

Fig. 11. Resilient Backpropagation topology using TAN, SIG and LIN functions.

Levenberg-Marquardt Backpropagation neural network tested using 3 different topologies based on hidden layers, 2 hidden layers with 15 neurons result $100 \%$ accuracy, while 3 hidden layers each with 15 neurons results $100 \%$ accuracy, 4 hidden layers with 15 neurons each results $80 \%$ accuracy, Fig. 12 shows the obtained results. The best result obtained from 2 and 3 hidden layers with 15 neurons each results $100 \%$ accuracy will be tested using different activation function SIG and LIN for performance evaluation, the best result is TAN function with $100 \%$ accuracy while SIG function produce 93\% and LIN produce 93\%, Fig. 13 shows the obtained results.

The only neural network detects the 15 persons with $100 \%$ is Levenberg-Marquardt, the $100 \%$ is obtained in more than one topology 2 and 3 hidden layers produce full detection of individuals, Fig. 13 shows the Final results of all neural network tested Levenberg, Resilient and Gradient, all output layers applied are 4-bit binary pattern, for top results from Fig. 14 more tests are to be examined to prove the stability of results on $\mathrm{N}$ number of persons rather than 15 . The purpose of getting such results may refer to this function is completely suitable for biological data.

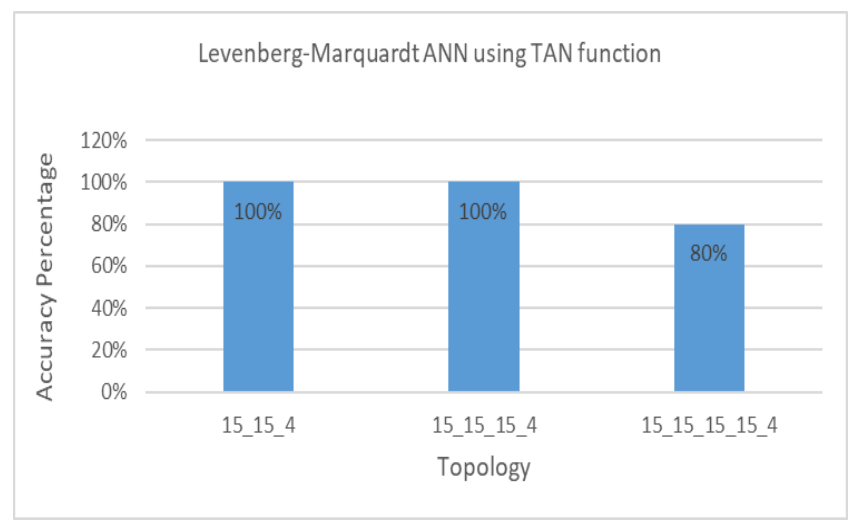

Fig. 12. Levenberg-Marquardt with 3 different topology accuracies using TAN function. 


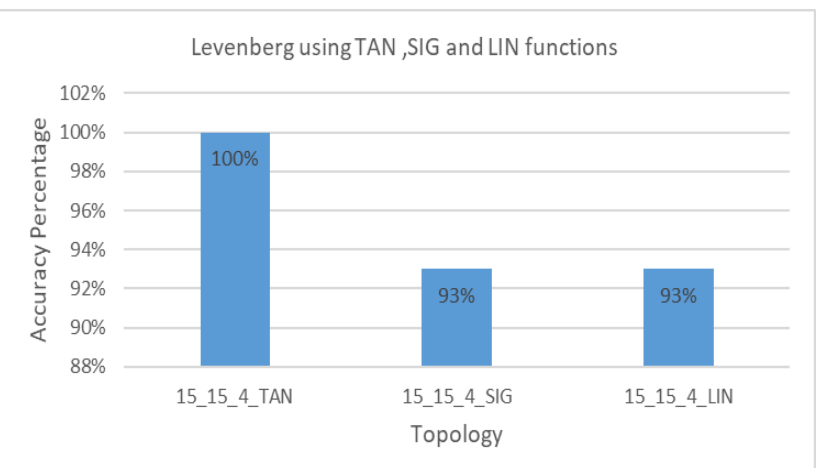

Fig. 13. Levenberg-Marquardt topology using TAN, SIG and LIN functions.

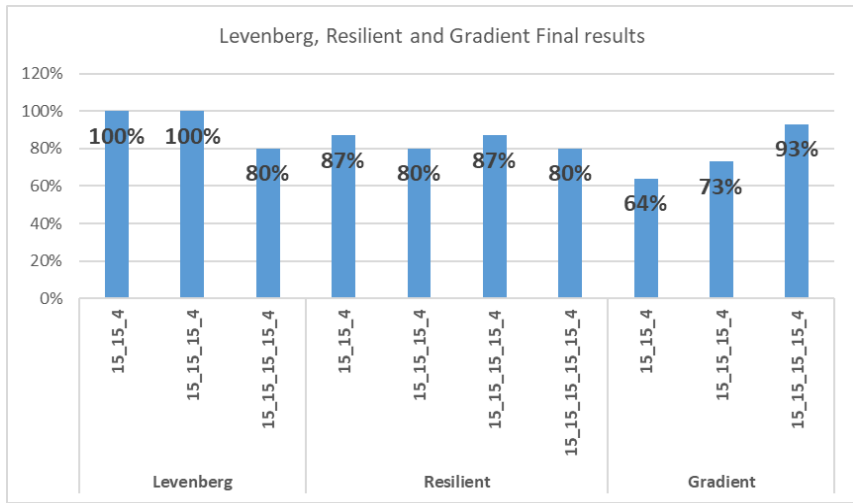

Fig. 14. Levenberg, Resilient and Gradient Final results

The only neural network that detects all the 15 persons with $100 \%$ is the one that uses the Levenberg-Marquardt, since the number of persons may increase which is needed to test the different output patterns. This is required in order to prove the stability and consistency of the performance accuracy of identifying individual person in case of large number of samples, 6-bit output pattern, 8-bit output pattern and 10-bit output pattern are applied and examined using Levenberg-Marquardt backpropagation.

The Levenberg-Marquardt Backpropagation neural network is tested using 4, 6, 8 and 10 binary output patterns, and based on the results obtained, 4-bit output produces $100 \%$ accuracy, 6-bit output produces $100 \%$ accuracy, 8-bit output produces $93 \%$ accuracy, and finally the 10-bit output produces $93 \%$ accuracy, Fig. 15 shows the obtained results.

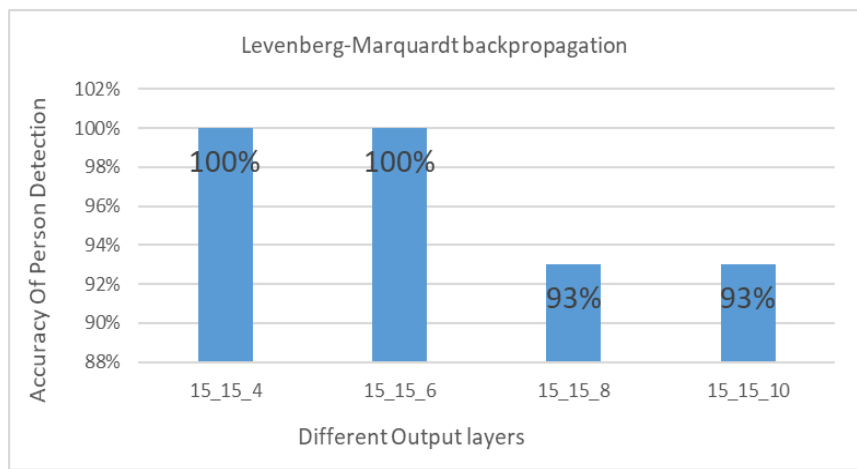

Fig. 15. 6 bit output pattern, 8 bit output pattern and 10 bit output pattern

Tables 3 and 4 show the performance accuracy percentage obtained from three different learning algorithms for three different neural net topologies and three different activation functions.

Table 3. The performance accuracy percentage found from 3 different learning algorithms for 3 different neural net topologies.

\begin{tabular}{lccc}
\hline Learning Algorithm & \multicolumn{3}{c}{ Performance Accuracy (\%) } \\
\cline { 2 - 4 } & 2 hidden & 3 hidden & 4 hidden \\
Resilient & $87 \%$ & $80 \%$ & $87 \%$ \\
Gradient Descent & $64 \%$ & $73 \%$ & $93 \%$ \\
Levenberg-Marquardt & $100 \%$ & $100 \%$ & $80 \%$ \\
\hline
\end{tabular}

Table 4. The performance accuracy percentage found from 3 different learning algorithms for 3 different Activation Functions.

\begin{tabular}{lccc}
\hline Learning Algorithm & \multicolumn{3}{c}{ Performance Accuracy (\%) } \\
\cline { 2 - 4 } & TAN & SIG & LIN \\
Resilient & $87 \%$ & $80 \%$ & $80 \%$ \\
Gradient Descent & $93 \%$ & $73 \%$ & $60 \%$ \\
Levenberg-Marquardt & $100 \%$ & $93 \%$ & $93 \%$ \\
\hline
\end{tabular}

\section{CONCLUSION}

This paper presents a study to measure the neural net classifier performance for human identification from odour based on algorithms of different learning employing three varying neural net topologies. The ideal human identification results are attained with the Levenberg-Marquardt backpropagation; the worse results for human identification are found from Gradient Descent backpropagation in some of the topologies. This paper also investigates the performance of three different activation functions TAN- Sigmoid transfer function, LOG- Sigmoid transfer function, and Linear transfer function. The best human identification results are obtained when the TAN- Sigmoid transfer function and the worse human identification results are obtained from Linear transfer function in some of the topologies. A study has been conducted to evaluate the performance of high number of persons identification on Levenberg-Marquardt backpropagation, the result obtains prove the rigidity and high accuracy of human detection in case of high number of persons.

\section{REFERENCES}

1. Sharma, Bhavna \& K. Venugopalan, Prof. (2014). Comparison of Neural Network Training Functions for Hematoma Classification in Brain CT Images. IOSR Journal of Computer Engineering. 16. 31-35. 10.9790/0661-16123135.

2. Priya, Saravana \& Mukesh, Rajeswari. (2019). Multimodal Biometric Authentication Using Back Propagation Artificial Neural Network. International journal of simulation: systems, science \& technology. 19. 10.5013/IJSSST.a.19.06.29.

3. W. F. Leung, S. H. Leung, W. H. Lau and A. Luk, "Fingerprint recognition using neural network," Neural Networks for Signal Processing Proceedings of the 1991 IEEE Workshop, Princeton, NJ, USA, 1991, pp. 226-235. doi: 10.1109/NNSP.1991.239519 
4. Erdogmus, Nesli \& Marcel, Sébastien. (2013). Spoofing in 2D face recognition with 3D masks and anti-spoofing with Kinect. IEEE 6th International Conference on Biometrics: Theory, Applications and Systems, BTAS 2013. 1-6. 10.1109/BTAS.2013.6712688.

5. Sabri A.Q., Alfred R. (2018) Evaluation of Artificial Neural Network in Classifying Human Gender Based on Odour. In: Alfred R., Iida H., Ag. Ibrahim A., Lim Y. (eds) Computational Science and Technology. ICCST 2017. Lecture Notes in Electrical Engineering, vol 488. Springer, Singapore

6. Shukla, Richa \& Shukla, Reenu \& Shukla, A \& Sharma, Sanjeev \& Tiwari, Nirupama. (2012). Gender Identification in Human Gait Using Neural Network. International Journal of Modern Education and Computer Science. 4. 70-75. 10.5815/ijmecs.2012.11.07.

7. Michelsanti, Daniel \& Ene, Andreea-Daniela \& Guichi, Yanis \& Stef, Rares \& Nasrollahi, Kamal \& Moeslund, Thomas. (2017). Fast Fingerprint Classification with Deep Neural Networks. 202-209. 10.5220/0006116502020209.

8. Marak, Pavol \& Hambalík, Alexander. (2016). Fingerprint Recognition System Using Artificial Neural Network as Feature Extractor: Design and Performance Evaluation. Tatra Mountains Mathematical Publications. 67. 10.1515/tmmp-2016-0035.

9. Elaydi, H \& Abukmeil, Mohanad \& Alhanjouri, Mohammed. (2013). Palmprint Recognition Using Multiscale Transform, Linear Discriminate Analysis, and Neural Network. Science Journal of Circuits, Systems and Signal Processing. 2. 112. 10.11648/j.cssp.20130205.13.

10. Manuel Ramírez-Cortés, Juan \& Gomez-Gil, Pilar \& Alarcon-Aquino, Vicente \& Baez, David \& Enriquez-Caldera, Rogerio. (2011). A Biometric System Based on Neural Networks and SVM Using Morphological Feature Extraction from Hand-Shape Images. Informatica, Lith. Acad. Sci.. 22. 225-240.

11. Wang, Ruxin \& Han, Congying \& Wu, Yanping \& Guo, Tiande. (2014). Fingerprint Classification Based on Depth Neural Network.

12. S. R. Patil and S. R. Suralkar, 'Fingerprint Classification using Artificial Neural Network', International Journal of Emerging Technology and Advanced Engineering, Volume 2, Issue 10, pp. 513-517, 2012, ISSN 2250-2459

13. N. Narang and T. Bourlai, "Gender and ethnicity classification using deep learning in heterogeneous face recognition," 2016 International Conference on Biometrics (ICB), Halmstad, 2016, pp. 1-8. doi: 10.1109/ICB.2016.7550082

14. S. N. Kohail, "Using artificial neural network for human age estimation based on facial images," 2012 International Conference on Innovations in Information Technology (IIT), Abu Dhabi, 2012, pp. 215-219. doi: 10.1109/INNOVATIONS.2012.6207735

15. Kalansuriya, Thakshila \& Dharmaratne, Anuja. (2015). Neural Network based Age and Gender Classification for Facial Images. International Journal on Advances in ICT for Emerging Regions (ICTer). 7. 10.4038/icter.v7i2.7154.

16. Sabri A.Q., Alfred R. (2018) Volatile Organic Compounds (VOCs) Feature Selection for Human Odor Classification. In: Alfred R., Iida H., Ag. Ibrahim A., Lim Y. (eds) Computational Science and Technology. ICCST 2017. Lecture Notes in Electrical Engineering, vol 488. Springer, Singapore

17. A. Voss, K. Witt, C. Fischer, S. Reulecke, W. Poitz, V. Kechagias, R. Surber, HR. Figulla, "Smelling heart failure from human skin odour with an electronic nose," Conf Proc IEEE Eng Med Biol Soc. 2012;2012:4034-7. doi: 10.1109/EMBC.2012.6346852.

18. Sunita Roy and Samir K. Bandyopadhyay, "Gender recognition using Self Organizing Map (SOM) - an unsupervised ANN approach," International Journal of Emerging Research in Management \&Technology, 2014.
19. Ramesha, K \& K B, Raja \& K R, Venugopal \& Patnaik, Lalit. (2010). Feature Extraction based Face Recognition, Gender and Age Classification. International Journal on Computer Science and Engineering. 2.

20. S. Omatu, "Odor classification by neural networks," 2013 IEEE 7th International Conference on Intelligent Data Acquisition and Advanced Computing Systems (IDAACS), Berlin, 2013, pp. 309-314. doi: 10.1109/IDAACS.2013.6662695

21. Chansri, Chana \& Srinonchat, Jakkree. (2010). Personal shirt odor classification using an electronic nose. 10.1109/ICCAE.2010.5451662.

22. Bassiouni, Mahmoud. (2016). A Machine Learning Technique for Person Identification using ECG Signals. IOSR Journal of Applied Physics. 1. 37.

23. Serdar Bascil, M \& Temurtas, Feyzullah. (2011). A Study on Hepatitis Disease Diagnosis Using Multilayer Neural Network with Levenberg Marquardt Training Algorithm. Journal of medical systems. 35. 433-6. 10.1007/s10916-009-9378-2.

24. Rehman M.Z., Nawi N.M. (2011) The Effect of Adaptive Momentum in Improving the Accuracy of Gradient Descent Back Propagation Algorithm on Classification Problems. In: Mohamad Zain J., Wan Mohd W.M.., El-Qawasmeh E. (eds) Software Engineering and Computer Systems. ICSECS 2011. Communications in Computer and Information Science, vol 179. Springer, Berlin, Heidelberg

25. Rehman Gillani, Syed Muhammad \& Mohd Nawi, Nazri. (2012). Studying the Effect of Adaptive Momentum in Improving the Accuracy of Gradient Descent Back Propagation Algorithm on Classification Problems. International Journal of Modern Physics: Conference Series. 9. 432-439. 10.1142/S201019451200551X.

26. Chien-Sheng, Chen \& Szu-Lin, Su. (2010). Resilient Back-propagation Neural Network for Approximation 2-D GDOP. Lecture Notes in Engineering and Computer Science. 2181.

27. Mohd Nawi, Nazri \& Khan, Abdullah \& Rehman Gillani, Syed Muhammad. (2013). A New Levenberg Marquardt Based Back Propagation Algorithm Trained with Cuckoo Search. Procedia Technology. 11. 10.1016/j.protcy.2013.12.157. Muaidi, Hasan. (2014). Levenberg-marquardt learning neural network for part-of-speech tagging of arabic sentences. WSEAS Transactions on Computers. 13. 300-309.

28. Lahmiri, Salim. (2011). A Comparative Study of Backpropagation Algorithms In Financial Prediction. International Journal of Computer Science, Engineering and Applications. 1. 15-21. 10.5121/ijcsea.2011.1402.

29. N. Nimsuk and T. Nakamoto, "Study of Odor Classification in Dynamically Changing Concentration using QCM Sensor Array and Short-Time Fourier Transform," TRANSDUCERS 2007 - 2007 International Solid-State Sensors, Actuators and Microsystems Conference, Lyon, 2007, pp. 2469-2472. doi: 10.1109/SENSOR.2007.4300671

30. He, Xiaochuan \& Wei, Shoushui \& Wang, Ruiqing. (2008). Independent Component Analysis and Neural Network Applied on Electronic Nose System. 490-493. 10.1109/ICBBE.2008.119.

31. C. Wongchoosuk, T. Youngrod, H. Phetmung, M. Lutz, T. Puntheeranurak and T. Kerdcharoen, "Identification of people from armpit odor region using networked electronic nose," 2011 Defense Science Research Conference and Expo (DSR), Singapore, 2011, pp. 1-4. doi: 10.1109/DSR.2011.6026826 


\section{AUTHORS PROFILE}

Ahmed Qusay Sabri is a lecturer at the Department of Information Technology, Community College, University of Sharjah, Sharjah-Khorfakkan, UAE. He obtained his master's degree from Saddam University, Iraq and Bachelor degree from Al. Mansour University College, Iraq

Rayner Alfred is an Associate Professor of Computer Science at the Faculty of Computing and Informatics, Universiti Malaysia Sabah in Malaysia that focuses on Data Science and Software Engineering programmes. He leads and defines projects around knowledge discovery, information retrieval and machine learning that focuses on building smarter mechanism that enables knowledge discovery in structured and unstructured data. His work addresses the challenges related to big data problem: How can we create and apply smarter collaborative knowledge discovery and machine learning technologies that bridge the structured and unstructured data mining and cope with the big data problem. Rayner completed his $\mathrm{PhD}$ in 2008 looking at intelligent techniques using machine learning to model and optimize the dynamic and distributed processes of knowledge discovery for structured and unstructured data. He holds a PhD degree in Computer Science from York University (United Kingdom), a master's degree in computer science from Western Michigan University, Kalamazoo (USA) and a Computer Science degree from Polytechnic University of Brooklyn, New York (USA) where he was the recipient of the Myron M. Rosenthal Academic Achievement Award for the outstanding academic achievement in Computer Science in 1994. He has authored and co-authored more than 100 journals/book chapters and conference papers, editorials, and served on the program and organizing committees of numerous national and international conferences and workshops. 